\title{
SAO 244567 (HEN1357) : A POST-AGB STAR WHICH HAS TURNED INTO A PLANETARY NEBULA WITHIN THE LAST 20 YEARS
}

\author{
M. PARTHASARATHY \\ Indian Institute of Astrophysics, Bangalore, India \\ partha@iiap.ernet.in
}

SAO 244567 (Hen1357) (IRAS 17119-5926) is a high galactic latitude $\left(1=331^{\circ}, \mathrm{b}=-12^{\circ}\right)$ early type star, originally classified as a B or A type H-alpha emission line star by Henize (1976). It is an IRAS source with far infrared colours similar to planetary nebulae. The IUE ultraviolet spectra obtained during the last eight years show that the central star is rapidly evolving. It is found that the central star of this young PN has faded by a factor of 3 within the last seven eight years. The terminal velocity of the stellar wind has decreased from $-3500 \mathrm{~km} / \mathrm{sec}$ in 1988 to almost

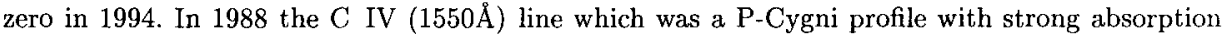
component had almost vanished by 1994 . The CIII] 1909A emission strength increased markedly within 4 years from 1988 to 1992 . The optical spectra obtained since 1990 shows very clearly only the nebular spectrum which is very similar to that of low excitation planetary nebula. The optical spectrum of SAO 244567 obtained in 1971 shows that it was a post-AGB B 1 or B2 supergiant at that time. This result shows that SAO 244567 has turned into a planetary nebula within the last 20 years. Recently Bobrowsky (1994) obtained narrowband optically resolved images in both H-beta and [OIII] 5007A with the HST planetary camera which revealed a well resolved nebula of size 2 seconds of arc. In this paper we discuss the recent new results. 